\title{
Die evangelikale Presse in der Bundesrepublik Deutschland*
}

\author{
von K. Rüdiger Durth
}

Trotz ihrer hohen Auflagen ist die evangelikale Presse bislang kein Gegenstand publizistischer Untersuchungen gewesen. Über ihre Wirksamkeit ist so gut wie nichts bekannt.

Wird mit der Einführung des Begriffs ,evangelikale Presse' nicht einer strukturellen Auseinanderentwicklung in verschiedene Arten von Publizistik innerhalb der Evangelischen Kirche in Deutschland Vorschub geleistet? Eine Frage, die nicht leichtfertig beantwortet werden kann. Denn der Dschungel evangelischer Presse verfügt zweifellos über manchen Wildwuchs, der förderungswürdigen Publikationen das Licht wegnimmt. Deshalb klingt das Wort Konzentration - im Gegensatz zur Situation der säkularen Massenmedien - in den Ohren vieler kirchlicher Medienexperten nach Rettung, Ausweg.

Doch einen Ausweg stellt die Konzentration um der Konzentration willen keineswegs dar, sondern würde zwangsläufig zu einer Versteppung der evangelischen Medienlandschaft führen. Aber der Ruf nach der einheitlichen evangelischen Stimme, etwa auf dem Nachrichtenmarkt, hat ja nicht unbedingt etwas mit Konzentration zu tun, sondern eher mit der durchaus wünschenswerten Einheit in Vielfalt, um so kirchlicher Massenkommunikation zu mehr Wirksamkeit in Kirche und Gesellschaft zu verhelfen.

Unbestritten ist, daß evangelikale Presse ein Bestandteil der evangelischen Publizistik ist und bleiben muß. Doch die vorfindliche Situation der protestantischen Kirchen und Gemeinschaften wird zu einem großen Teil von den Evangelikalen geprägt. Gleiches gilt für die Presse. Allen Einheitsforderungen zum Trotz kann nicht verleugnet werden, daß es zwei Gruppen gibt, nämlich eine, die man als kirchen-offizielle Presse bezeichnen kann, ohne ihr deshalb die journalistische Selbständigkeit zu bestreiten, und die andere, die weitgehend auf Spenden angewiesen ist.

\section{Was ist evangelikal?}

Die evangelikale Massenkommunikation hat noch keinen Eingang in die Publizistikwissenschaft gefunden, auch nicht in die kirchliche Medienarbeit. Sie wird im Protestantismus weitgehend als Bestandteil der evangelischen Presse betrachtet, so u.a. in der „Bestandsaufnahme evangelischer Periodika“ aus dem Jahr $1976^{1}$ oder im „Publizistischen Gesamtplan“ der Evangelischen Kirche in Deutschland (EKD) von $1979^{2}$.

Selbst innerhalb der evangelikalen Bewegung wächst erst langsam das Bewußtsein von der Notwendigkeit einer eigenen Massenkommunikation. Im „Evangelischen Gemeindelexikon"3, das als repräsentatives Nachschlagewerk für die deutschen Evangelikalen gelten darf, finden sich weder die Stichworte Medien, Massenmedien, Massenkommunikation noch evangelikale Presse oder Publizistik. Das

K. Rüdiger Durth ist Parlamentsredakteur der „Kölnischen/Bonner Rundschau“ in Bonn, zugleich (nebenamtlicher) Pastor der Evangelischen Kirche im Rheinland. 
Thema wird versteckt abgehandelt unter „Erbauungsschrifttum“ und „Zeitschriften“.

Die Ursache für dieses Defizit ist vielfältig, wird aber langsam erkannt. Obwohl die Evangelikalen über ein umfangreiches Buch- und Traktatprogramm verfügen, standen (und stehen) sie der Massenkommunikation und deren Voraussetzungen in der heutigen Zeit skeptisch gegenüber. Man beschäftigte sich kaum mit ihr. Das ändert sich langsam, wobei dem 1971 ins Leben gerufenen „Informationsdienst der Deutschen Evangelischen Allianz“, Signum „idea“, eine wesentliche Rolle zufällt.

Die Schwierigkeit beginnt bereits mit dem Begriff „,evangelikal“, „der zum gegenwärtigen Zeitpunkt ausgesprochen schwierig zu definieren ist ${ }^{\star 4}$, obwohl damit gerechnet werden kann, daß sich bis zu rund 40 Prozent der evangelischen Christen in der Bundesrepublik Deutschland im weiteren Sinn zu den Evangelikalen zählen.

Das Wort ist aus dem anglo-amerikanischen Sprachraum übernommen worden und hat sich im deutschen Sprachraum seit Ende der 50er Jahre durchgesetzt und umfaßt vor allem Gruppen des Pietismus, der Erweckungs- und Gemeinschaftsbewegung, der amerikanischen Evangelisations-Arbeit, aber auch fundamentalistische und konservative protestantische Richtungen. In den Massenmedien wird ,evangelikal' pauschal als Sammelbegriff für alles verwandt, was nicht offiziell landeskirchlich ist, und damit wird der Sache kein guter Dienst erwiesen.

Ulrich Betz weist darauf hin, daß der Begriff, evangelikal' ,, von einem bunten Spektrum kirchlicher, freikirchlicher und überkirchlicher Gruppen und Gruppierungen für sich in Anspruch genommen wird, die theologisch voneinander abweichen"s. Mit Betz jedoch können folgende gemeinsame vier Merkmale zur Verständigung über das, was evangelikal ist, beitragen:

1. Die persönliche Erfahrung der Errettung durch Christus, der Empfang der Vergebung der Sünden und die Gewißheit des Heils im Glauben.

2. Zusammengehörigkeit mit allen Menschen, die von Herzen Jesus Christus nachfolgen.

3. Die Bereitschaft, sich persönlich in Evangelisation und Mission zu engagieren.

4. Die verpflichtende Bindung an die Bibel als das Wort Gottes ${ }^{6}$.

Kurt Heimbucher unterscheidet Bekenntnis-Evangelikale (kirchliches Bekenntnis

- Autorität), Pfingst-Evangelikale (Charismatiker) und Allianz-Evangelikale (Pietismus, Erweckungsbewegung).

Die aufgezeigten Schwierigkeiten einer genauen Definition von „evangelikal“ machen deutlich, wie schwer erst eine Begriffsbestimmung für „evangelikale Massenkommunikation" ist. Denn:

1. Vor allem die Bekenntnis- und Allianz-Evangelikalen nach Heimbucher verstehen sich als Glieder der evangelischen Kirche. Deshalb wird man ihre Publizistik auch als evangelische bezeichnen können.

Zahlreiche evangelikale Publikationen wehren sich ausdrücklich gegen diese „Klassifizierung“, um ihrerseits Polarisationen zu vermeiden.

2. Viele Bestsellerautoren wie Jörg Zink haben ihre Leserschaft im gesamten protestantischen Spektrum und darüber hinaus, während Gerhard Bergmann oder Christa Meves eindeutig der evangelikalen Bewegung zuzuordnen sind. 
3. Die Traktatliteratur, die seit einem Jahrzehnt ungewohnte Auflagen erreicht, wird zwar weitgehend von evangelikalen Verlagen herausgegeben, aber von allen kirchlichen Gruppen gelesen.

4. Was für das Traktat gilt, gilt auch für die Erbauungsliteratur - vom Abreißkalender bis zum Losungsbuch, vom Roman bis zur christlichen Biographie.

\section{Die zzeeite Agentur - „idea“}

Bis vor wenigen Jahren war „idea“ - Informationsdienst der Evangelischen Allianz - fast ausschließlich im innerkirchlichen Bereich bekannt und galt als Nachrichtenagentur für die evangelikale Publizistik. Inzwischen ist „idea“, 1970 gegründet, zu einem Nachrichtendienst für den gesamten Protestantismus geworden. Damit trat er notwendigerweise in Konkurrenz zum Evangelischen Pressedienst (epd).

Die Anfänge waren bescheiden. Gründer Horst Marquardt, hauptamtlicher Programmdirektor des „Evangeliumsrundfunks“, wollte der evangelikalen Bewegung und Publizistik ein Organ zur Seite stellen, das Meldungen, Berichte und Meinungen verbreitete, die in epd nicht oder nur unzureichend Aufnahme fanden. Aus organisatorischen Gründen wurde Wetzlar Redaktionssitz - die räumliche Nähe zum ,Evangeliumsrundfunk' machte dies notwendig.

Der Redaktion ist der Vorstand unter Leitung von Horst Marquardt vorgesetzt, der wiederum dem idea-Verein (1981 rund 30 Mitglieder aus Freikirchen, evangelikalen Werken und Journalisten) veranwortlich ist. Zunächst erschien „idea“ unregelmäßig bei nebenamtlicher Leitung. 1974 wurde der erste hauptamtliche Redakteur berufen, 1977 der zweite. Nach Rolf Hille und Ingrid Kastellan wurde 1978 Helmut Matthis zum verantwortlichen Redakteur berufen, der von Wolfgang Thielmann vertreten wird.

Die ,idea'-Redakteure kamen nicht aus dem Journalismus, waren also keine „Profis", sondern hatten meist eine theologische Ausbildung absolviert. Mit einfachsten redaktionellen Mitteln gelang ihnen der stetige Ausbau des Nachrichtendienstes, der laut Eigenwerbung „zu einer wichtigen Stimme im deutschen Protestantismus geworden" ist.

Erschien ,idea“ bis 1980 einmal wöchentlich (montags), so wird er seit August 1980 als „Basisdienst“ zusätzlich noch donnerstags herausgegeben. Er wendet sich vor allem an die Redaktionen der Tageszeitungen und Rundfunkanstalten, die Kirchenpresse und interessierte kirchliche Persönlichkeiten, vor allem solche in leitender Position.

Wurde er bis 1979 an alle Interessenten verschickt, so ist für diese ,idea-spektrum“ gedacht, das (in der Regel 16 Seiten) eine Auswahl der wichtigsten Nachrichten, Berichte, Interviews und Kommentare der beiden Basis-Ausgaben pro Woche enthält und als „Nachrichten und Meinungen aus der evangelischen Welt“(Untertitel) zu einer Zeitschrift geworden ist, die mit dem epd-Nachrichtenspiegel ,evangelische Information" konkurriert, auflagenmäßig diesen jedoch überholt hat.

Bereits auf der „idea“-Vorstandssitzung vom Dezember 1979 konnte Marquardt mitteilen, da $B$ die Basis-Ausgabe 820 Abonnenten (epd zentral 700) habe, „ideaspektrum“ 3.000 („Der besondere Akzent evangelikaler Publizistik“) im Gegensatz zu 2.000 Abonnenten für „evangelische Information“. 1980 überschritt „spektrum“ 
bereits die 4.000er Grenze, obwohl einige evangelische Kirchenzeitungen eine bezahlte Werbeanzeige ablehnten, u.a. die „Niedersächsische Evangelische Zeitung “ und die westfälische „Unsere Kirche“ (beide im November 1979).

Nach wie vor sieht die Evangelische Kirche in Deutschland in „idea“ ein „Richtungsorgan", das soll heißen: ein Organ der evangelikalen Bewegung. Aus diesem Stadium freilich ist dieser Nachrichtendienst längst entwachsen - und das brachte ihm auch den Konflikt mit epd.

In der allgemeinen Publizistik findet „idea“ zunehmend Interesse und Abdrucke, u.a. auch in den überregionalen Zeitungen. Die Redaktion (mit nur zwei hauptamtlichen Redakteuren besetzt) nimmt längst alle großen EKD- und Landeskirchlichen Veranstaltungen selbst wahr, berichtet hin und wieder auch aus der katholischen Welt und verfügt über gute ökumenische Informationsquellen.

Das Schwergewicht der Nachrichtenarbeit von „idea“ liegt auf Informationen aus der evangelikalen Welt, den Freikirchen, der Evangelisations- und Missionsarbeit. Aber die Amtskirche hat in den Nachrichten längst einen festen Platz, und im Berichts- und Kommentarteil kommen leitende Bischöfe in eigenen Beiträgen oder in Interviews ebenso zu Wort wie unterschiedlichste Mitarbeiter aus dem Inund Ausland. In zahlreichen Kirchenkanzleien gilt „idea“ längst als unverzichtbar, was aber nicht öffentlich gesagt wird.

Abonnenten hat ,idea“ "nach eigenen Angaben in rund 50 Ländern. Die wichtigsten Nachrichten werden auch zu einer englisch-sprachigen Ausgabe zusammengefaßt, die vor allem für den Bereich der Ökumene, England und die USA bestimmt ist.

Das einstige „Richtungsorgan“, das zugleich politisch der CDU nahestand, wurde nach eigenem Verständnis zu einer „Brücke zwischen Evangelikalen untereinander, den Landes- und Freikirchen sowie den Evangelikalen und anderen Gruppen“. Auch politisch hat eine merkliche Auflockerung stattgefunden, die neue Leserschichten erschlossen hat und zu einem größeren Interesse bei den Massenmedien führte.

Ergänzt wird das „idea“-Nachrichtenangebot neuerdings noch durch die „idea-Dokumentation" wichtiger kirchlicher Reden oder Ereignisse im Wortlaut.

\section{Der mißglückte Versuch}

„Die Deutsche Evangelikale Zeitung (DEZ) kommt“, versprach 1979 der Evangelist Günter Tesch aus dem nordheidischen Buchholz in seiner „Stimme des Evangeliums“, die zugunsten der neuen Wochenzeitung mit unverkennbaren graphischen Merkmalen der „Deutschen Zeitung/Christ und Welt" eingestellt wurde. DEZ wollte ,über Sorgen, Nöte und Freuden unterrichten und davon erzählen, wie die Gemeinde der Christen in der Welt lebt, vorankommt, denkt und spricht ${ }^{47}$. Tesch wollte ,zur Orientierung in einer verworrenen Zeit verhelfen ${ }^{\text {"8 }}$ und ging bald auf eigenen Irrwegen unter.

Der Hauptvorstand der Deutschen Allianz distanzierte sich bereits im Vorfeld der Gründung von dieser Zeitung, laut Eigenwerbung „der Schlüssel für qualifizierte evangelikale Information", Begründung: Der Herausgeber hatte die Deutsche Allianz nicht konsultiert und auch nicht versucht, eine „breite Basis“ unter den Evangelikalen herzustellen ${ }^{9}$. Nach einer Pilotnummer Ende Februar 1979 mit 
20.000 Exemplaren erschien DEZ ab 28. März alle zwei Wochen mit 16 Seiten Umfang zum Preis von 2,- DM.

Vergessen schienen schon damals die lauten Worte von Tesch zu sein, der 1972 das „Missionswerk Evangelistic Team e.V.“ gegründet hatte. Im Herbst 1978 nämlich war noch die Rede von 250.000 Exemplaren Startauflage ${ }^{10}$, einer gesicherten finanziellen Basis. Nach Tesch zielte DEZ auf die „konservativen Protestanten“, die bislang noch von keiner Wochenzeitung angesprochen wurden. Das Blatt wolle sich von „evangelikalen Positionen“ aus auch zu politischen, wirtschaftlichen und kulturellen Themen äußern ${ }^{11}$.

Während Tesch, der schon immer wegen unorthodoxer Evangelisation umstritten war, noch verkündete, „Qualität setzt sich durch“ und seine Zeitung als „die bessere Alternative" anpries, stellt ihm Otmar Schulz im Evangelischen Pressedienst (epd) ein vernichtendes Zeugnis aus: „Um Klassen“ seien die Wochenzeitschriften der Freikirchen dieser „Hauspostille des volksmissionarischen Einzelgängers“ überlegen. Schulz machte ein „Landsmannschaftsdeutsch" aus und ahnte die Reizworte von DEZ, nämlich kommunistisch, linkslastig, ökumenisch. In zwei Dingen freilich irrte Schulz. Er war überzeugt, daß es dieser Zeitung an Geld nicht mangeln würde und daß man „noch eine Weile damit leben" müsse ${ }^{12}$. Aber die Zeitschrift kam nicht an. Es folgten harte öffentliche Auseinandersetzungen zwischen der Deutschen Allianz, idea und der DEZ, die zu Strafanzeigen führten. Die Auflage sank, die Zeitung wurde in „Deutsche Evangelische Rundschau“ umbenannt und mußte trotz der Berufung von Dieter Kraeter (Rheinischer Merkur) zum Chefredakteur im Mai $1980 \mathrm{ihr}$ Erscheinen einstellen.

Scheiterte die „Deutsche Evangelikale Zeitung“ nur wegen Tesch? Die Frage hat viele im evangelikalen Lager bewegt, die zunächst dem Experiment Wochenzeitung durchaus positiv gegenüberstanden und von dem Streit um den Herausgeber zunächst verschont blieben. Die Deutsche Allianz fürchtete zu Recht, daß ein offener Konflikt vor allem der evangelikalen Bewegung angelastet werde und das Vertrauen in die vielen auf Spenden angewiesene Werke und Gruppen zerstöre. Erst als Tesch unverhohlen seine "Gegner" in der DEZ angriff, ging die Deutsche Allianz zur öffentlichen Distanzierung über.

Im Blick auf die Zeitung selbst waren jedoch andere G ründe wichtiger: Eine Zweiwochen-Publikation mit 16 Seiten im Zeitungsformat erfordert eine klare Zielgruppe, die pauschal mit „evangelikal“ nicht ausreichend definiert ist. Sie erfordert angesichts bundesweiter Verbreitung, Anzeigengeschäft, Abonnementwerbung und -betreuung sowie durcktechnischer Probleme einen versierten Herausgeber, der etwas vom „Geschäft“ versteht und als Verleger voll ausgelastet ist.

\section{Die unbekannten Millionen}

Knapp 33 Millionen Exemplare pro Jahr beträgt die Auflage von 144 evangelikalen Zeitschriften. Den größten Anteil davon haben 20 Verteilblätter mit fast 11 Millionen Exemplaren Jahresauflage. Gefolgt an dritter Stelle von den ihnen nahe verwandten Erbauungszeitschriften, von denen alle drei es auf eine Jahresauflage von über vier Millionen Exemplaren bringen.

Diese beiden Zeitschriftengruppen bilden zugleich die größte Unbekannte unter den „unbekannten Millionen“, weil es sehr schwer ist, ihre Zahl auch nur an- 
nähernd zu ermitteln. Zieht man in Betracht, daß viele Verlage Verteilblätter herausgeben, aber auch uns bekannte keine Angaben über ihre Auflagenhöhe und Zahl machen wollten, dann geht man nicht fehl, die Zahl der Verteil- und Erbauungs-Zeitschriften, die zur evangelikalen Publizistik zählen, mit 50 als Minimum anzugeben. Wahrscheinlich aber sind es erheblich mehr.

Gleiches trifft für die Missionszeitschriften zu, die mit 33 Titeln den größten Einzelteil in der Gruppe der 144 hier zugrundegelegten Zeitschriften ausmachen. Sie erreichen eine Jahresauflage von über 4,6 Millionen Exemplaren. Gleiche Erfahrung wie bei den Verteilblättern: Vor allem die kleineren evangelikalen Missionswerke zeigten sich nicht bereit, Angaben über ihre Publikationen zu machen. Zieht man die kleineren als Vergleich heran, die auf unsere Umfrage antworteten, und rechnet man die einschließlich der „schweigenden“ Verteil- und Erbauungszeitschriften hoch, dann dürfte sich eine evangelikale Jahresauflage von Zeitschriften ergeben, die bei mindestens 50 Millionen Exemplaren liegt.

\section{Gesamtübersicht über Zahl und Auflage der evangelikalen Presse}

\begin{tabular}{|l|c|c|c|r|}
\hline & Zahl & $\begin{array}{c}\text { Seiten } \\
\text { pro Ausgabe }\end{array}$ & $\begin{array}{c}\text { Auflage } \\
\text { pro Ausgabe }\end{array}$ & Jahresauflage \\
\hline Gemeinde-Zeitschriften & 11 & 212 & 80.450 & 3.125 .400 \\
Gruppen-Zeitschriften & 20 & 388 & 120.700 & 934.400 \\
Jugend-Zeitschriften & 19 & 466 & 246.400 & 3.789 .600 \\
Missions-Zeitschriften & 33 & 518 & 630.700 & 4.631 .000 \\
Theologische Zeitschriften & 15 & 433 & 190.200 & 861.400 \\
Mitarbeiter-Zeitschriften & 15 & 448 & 90.200 & 520.200 \\
Erbauungs-Zeitschriften & 3 & 15 & 182.100 & 4.105 .200 \\
Verteil-Zeitschriften & 20 & 120 & 935.000 & 10.700 .000 \\
Studenten-Zeitschriften & 2 & 88 & 6.500 & 26.000 \\
Verschiedene & 6 & 76 & 328.800 & 3.906 .000 \\
Zeitschriften & 144 & 2.764 & 2.811 .050 & 32.608 .200 \\
\hline Gesamt: & & &
\end{tabular}

Die evangelikale Presse als Bestandteil der protestantischen Presse ist bislang zu einem großen Teil überhaupt nicht erfaßt, was die 1978 erschienene Presseübersicht des Gemeinschaftswerks der Evangelischen Publizistik (GEP) zeigt ${ }^{13}$.

Diese geht von wahrscheinlich 700 bis 800 Titeln für alle Periodika aus, in den erfaßten weit weniger Titeln sind auch solche aufgenommen, die z.T. nur örtlich erscheinen. Zieht man diesen Bogen, dürfte die Titelzahl weit über 1.000 (ohne Gemeindebrief) gehen. Das verändert dann auch die von GEP in dieser Untersuchung für wahrscheinlich gehaltene Auflage von rund 160 Millionen Exemplaren pro Jahr.

Die bereits dargestellten Probleme, die evangelikale Zeitschriftenpublizistik auch nur halbwegs vollständig aufzeigen zu wollen, verdeutlichen, wie schwer es ist, 
eine exakte Statistik zu erstellen, die die gesamte protestantische Presse umfaßt. Die hier aufgezeigten „unbekannten Millionen“ der evangelikalen Publizistik, die nur zum geringen Teil in den GEP genannten 160 Millionen Exemplaren Jahresauflage enthalten sind, verdeutlichen zugleich, wie groß der Anteil der evangelikalen Publizistik an der gesamtprotestantischen Presse ist.

\section{Gemeinde-Zeitschriften}

Die Gemeindezeitschriften entsprechen den landeskirchlichen Wochenzeitungen, ohne deren Auflagen zu erreichen. Das ist auch verständlich, da die Freikirchen über erheblich weniger Mitglieder verfügen. Das wirkt sich auch auf die Erscheinungsweise aus.

Immerhin erschienen vier der 11 Gemeindezeitschriften wöchentlich mit einer Gesamtauflage von 60.000 Exemplaren (im Jahr 2.880.000 Exemplaren).

Gemeinde-Zeitschriften im Überblick:

\begin{tabular}{|l|l|r|}
\hline \multicolumn{1}{|c|}{ Titel } & \multicolumn{1}{|c|}{ Gemeinde } & Auflage \\
\hline $\begin{array}{l}\text { Die Botschaft } \\
\text { Die Gemeinde }\end{array}$ & $\begin{array}{l}\text { Monatszeitschrift der Brüdergemeinden } \\
\text { Bund Evangelisch-Freikirchliche Gemeinden } \\
\text { Gemeinde } \\
\text { unterwegs }\end{array}$ & 3.000 \\
Der Gärtner & Mennonisten) & 16.700 \\
& Zeitschrift des Bundes Freier evangelischer & 1.200 \\
Heilszeugnisse & Gemeinden & 10.800 \\
Der Kriegsruf & Gemeinschaftsverbandes Mülheim-Ruhr & 3.000 \\
Missionsblatt & Die Zeitschrift der Heilsarmee in Deutschland & $17-18.000$ \\
Der Quäker & Mission Evangelisch-Lutherischer Freikirchen & 3.800 \\
Wegweisung & Monatsschrift der deutschen Freunde & $600-700$ \\
Wort und Geist & Monatszeitschrift der freien Brüder & 3.800 \\
Wort und Weg & Monatsschrift pfingstlicher Gemeinden & 5.000 \\
& Sonntagsblatt der Evangelisch-methodistischen & 14.500 \\
\hline
\end{tabular}

Sieben Zeitschriften erscheinen monatlich mit einer Auflage von 20.450 Exemplaren (im Jahr 245.400). Hier handelt es sich vor allem um die kleinsten der Freikirchen.

Mit einer Gesamtauflage von über 3,1 Millionen Exemplaren pro Jahr erreichen diese Gemeindezeitschriften dennoch eine beachtliche Auflage.

\section{Die nebenamtlichen Redaktionen}

Die evangelikale Publizistik zählt mit Sicherheit zu den preiswertesten Zeitschriften - geht man von dem Abonnement- oder erwünschten Spendenpreis aus. Das ergibt die Auswertung der Fragebogen von 70 evangelikalen Printmedien. Diese Untersuchung beansprucht nicht, nach den publizistisch-wissenschaftlichen 
Grundsätzen vorgenommen worden zu sein. Da sie jedoch Zeitschriften aller Typen und auch Verteilblätter enthält, kann sie für die evangelikale Publizistik als durchaus repräsentativ gelten. Vor allem gibt sie einen interessanten AufschluB über Gemeinsamkeiten und Unterschiede.

Die Angaben können nicht jeweils auf 100 Prozent hochgerechnet werden, da die befragten Zeitschriften-Redaktionen bzw. Verlage nicht alle den Fragenbogen vollständig ausfüllten. Vielfach wollte man sich nicht in die eigenen Karten schauen lassen. Oft konnte man leicht feststellen, da $B$ es sich um das Übersehen einer Frage handelte oder - was nicht selten war - die Rubriken mit einem Fragezeichen versehen waren. Schließlich haben sich zahlreiche Mitarbeiter von evangelikalen Zeitschriften kaum oder nur unzureichend mit der Situation ihrer eigenen Publikationen befaßt.

Das wird verständlich, wenn man bedenkt, daß über die Hälfte der hier ausgewerteten 70 Zeitschriften nicht von einem hauptberuflichen Redakteur geleitet werden. Das gilt keineswegs nur für die meisten Missions-Zeischriften, sondern auch für zahlreiche theologische auflagenstarke Periodika der unterschiedlichsten Gruppen. Nur wenige Zeitschriften geben an, über zehn und mehr Mitarbeiter zu verfügen. Die meisten Zeitschriften werden von weniger als fünf Mitarbeitern gestaltet, wobei die Zahlen 1 bis 3 am häufigsten genannt werden. Verfügt eine Redaktion über eine hauptamtliche Redaktionsleitung, dann beschränkt sich die Zahl der Mitarbeiter meist auf einen oder zwei.

Bedenkt man, daß die meisten dieser Mitarbeiter aus der evangelikalen Gemeindeoder Missionsarbeit kommen, sich in der betreffenden Gruppe bewährt oder ganz einfach Lust am Schreiben haben, dann kann man sich nur immer wieder von neuem wundern, welche Leistungen auf publizistischem Gebiet innerhalb der evangelikalen Bewegung erbracht werden. Eine hauptamtliche Redaktionsleitung besagt aber längst nicht, daß diese nur eine Zeitschrift betreut, sondern vielfach werden von ihr mehrere Verlagsprojekte redigiert.

Die überwiegende Zahl evangelikaler Zeitschriften erscheint in den Formaten DIN A 4 und DIN A 5, wobei letzteres die Mehrzahl bildet. Längst bedient sich die evangelikale Publizistik der modernen Druckmöglichkeiten, die sich aus den preiswerten Offsetverfahren ergeben und auch die Verwendung von Farbe ermöglichen. Das hektografierte Blatt bildet heute die große Ausnahme. Gleiches gilt mehr oder weniger für den Buchdruck.

Schaut man sich die Erscheinungsjahrgänge der evangelikalen Zeitschriften an, so fällt das zum Teil sehr junge Alter auf - bis zu 25 Jahren, wobei die unter zehn Jahren einen besonders großen Anteil haben. Dies dürfte mit Sicherheit auch im Zusammenhang mit den neuen Drucktechniken stehen, die Mut zu neuen Zeitschriften gaben. $\mathrm{Zu}$ den ältesten Zeitschriften, die zum Teil ins vergangene Jahrhundert zurückreichen, gehören vor allem die Gemeinde-Zeitschriften und einige Verteilblätter. Manche haben auch den Titel und die Zielgruppe im Lauf der Zeit geändert. Konkret bedeutet dies, daß der evangelikalen Publizistik durch die Revolution auf dem Sektor der Klein-Drucktechnik, die heute auch hohe Auflagen ermöglicht, bislang die Vielfalt der Zeitschriftentitel ermöglicht wird.

Dies belegen weiter auch diese Zahlen: Die meisten Redaktionen/Verlage geben an, ihre Zeitschrift ohne Zuschüsse herausgeben zu können. Auf dem finanziellen 
Sektor ist es am schwierigsten, gültige Aussagen zu machen, weil hier die Zurückhaltung von Angaben am größten ist. Auch ist es fast unmöglich, einen Durchschnittspreis anzugeben, weil die Finanzierung zu unterschiedlich ist.

- Die Gemeinde-Zeitschriften werden in der Regel im Abonnement vertrieben. Aufgrund der kleinen Zahlengröße der Gemeindeglieder ergibt sich für diese „Mitgliederpublizistik“ eine relativ niedrige Auflage. Das schlägt sich zwangsläufig im Bezugspreis nieder, der unter allen evangelikalen Zeitschriften-Typen am höchsten ist und im Jahr bis über $60 \mathrm{DM}$ betragen kann. Darin liegt wohl auch begründet, daß für diese Gruppe öfters eine sinkende Auflage angegeben wird, größere Zuwachsraten nicht zu erwarten sind.

- Die Missions-Zeitschriften erfüllen meist nicht nur die Funktion der Information von Freunden des jeweiligen Missionswerkes, sondern sind unverzichtbar für die Spendenwerbung. Deshalb werden diese Blätter kostenlos abgegeben und finanziert durch das einkommende Spendenaufkommen. Das erfordert eine hohe Auflage, nicht zuletzt deshalb, weil die Adressen potentieller Spender oft von unterschiedlichen Missionswerken angeschrieben werden. Einige dieser Blätter erbitten auch einen Jahresbezugspreis von wenigen Mark, kaum höher als 10 DM, oder geben die Herstellungskosten für den Jahresbezug als Spenden-Richtlinie an.

- Jugend- und Studentenpresse sind ebenfalls im Abonnementpreis sehr günstig. Dies wird zum Teil durch Verlagszuschüsse, zum Teil durch hohe Auflagen erreicht. Würde man die Kosten sehr hoch ansetzen, müßten die Verlage wohl mit Auflagen-Einbußen rechnen, weil es sich bei der Zielgruppe um Menschen mit niedrigen Einkommen handelt.

- Aber selbst anspruchsvolle Zeitschriften - vom redaktionellen Angebot bis zur graphischen Gestaltung und Druckqualität - versuchen; den Bezugspreis gering zu halten, um eine möglichst breite Streuung zu erreichen.

- Die Kosten der meisten Mitglieder-Zeitschriften gelten durch den monatlichen Mitgliedsbeitrag als abgegolten oder werden ebenfalls relativ niedrig angesetzt. Ein Verfahren, das auch in den säkularen Verbänden in der Regel angewandt wird, weil die regelmäßige Zeitschrift als Informations- und Anregungsmaterial für die praktische Arbeit für die Gruppe, das Werk unverzichtbar ist. Nicht zuletzt um den inneren Zusammenhalt zu fördern.

- Die Verteilblätter müssen zwangsläufig billig sein, um ihre Funktion erfüllen zu können. Entweder werden minimale Abonnentengebühren erhoben, oder es wird kostenlos verteilt. Hier spielen die Spenden ebenfalls eine nicht zu unterschätzende Rolle.

Insgesamt kann für die evangelikale Publizistik gesagt werden, daß sie auch in der Herstellung nicht sonderlich teuer kommt. Neben den bereits erwähnten Möglichkeiten durch neue Drucktechniken ist in der Regel der redaktionelle Aufwand bescheiden. Bei nebenamtlichen Redaktionsleitungen entfallen Gehälter und die ständig steigenden Sozialkosten. Selbst wo eine Zeitschriftenredaktion hauptamtlich vorhanden ist, bleibt die Zahl der Redakteure sehr niedrig. Kaum mehr als ein oder zwei Kräfte. Gleichzeitig arbeiten auch die meisten Mitarbeiter ehrenamtlich, erhalten also keine oder nur sehr bescheidene Honorare. 


\section{Der besondere Stil}

$\mathrm{Zu}$ den Besonderheiten der evangelikalen Publizistik zählt der ,missionarische Stil“. In der aktuellen säkularen Massenkommunikation wird - leider mit nachlassendem Erfolg - eine Trennung zwischen tatsachen-orientierten und meinungsbildenden Stilformen vorgenommen. Damit ist gemeint, daß Nachrichten und Meinungen nicht miteinander vermischt werden. Dies entspricht der angelsächsischen Tradition: "facts are sacred but comment is free" (Tatsachen sind unantastbar, Kommentierung jedoch ist freigestellt).

$\mathrm{Zu}$ den tatsachenorientierten Stilformen zählen publizistik-wissenschaftlich Meldung (auch unscharf Nachricht genannt, weil die Nachricht den Inhalt der Meldung bildet), Bericht, Interview, Reportage. Die meinungsorientierten Stilformen setzen sich zusammen aus Leitartikel, Kommentar, Rezension, Glosse, Karikatur. Seit einigen Jahren beobachten wir eine zunehmende Tendenz zum Hintergrundbericht, eine Art Mischung von Bericht und Kommentar. Diese Stilform wird vor allem von den Wochenzeitungen und Magazinen gepflegt.

Die evangelikale Presse unterscheidet weithin nicht zwischen den einzelnen journalistischen Stilformen, sondern verwendet in erster Linie den kommentierenden Bericht, man könnte auch das alte Wort vom „Artikel“ oder „Aufsatz“ verwenden.

Kennzeichnend ist für die Mehrzahl der Zeitschriften - die Gemeinde-, Verschiedene- und Theologische Zeitschriften bleiben hier unberücksichtigt - der missionarische Stil. Meint dieser in den säkularen Massenkommunikationsmitteln etwas Negatives, nämlich um politischer oder ideologischer Ziele willen bewußt gewählte Form, so zählt er in der evangelikalen Publizistik zum beherrschenden Schreibstil.

Allerdings nicht, um Politik zu „verkaufen“, sondern um den Leser für die Sache des Evangeliums zu gewinnen. Dieser Stil findet sich nicht nur in den persönlichen Worten der Schriftleiter, Missionsdirektoren etc. für den Leser - die vielen Zeitschriften einen Charakter der Briefzeitung geben (die übrigens in früherer Zeit sehr verbreitet war), sondern auch in den Reportagen, kirchlichen oder missionarischen Berichten. Dagegen fehlen weithin Nachrichten, knappe, informative Berichte, sachliche Interviews und Reportagen, die über den „Insider“ hinaus wirken wollen.

Hier setzt nicht nur die säkulare journalistische Kritik an der evangelikalen Presse ein, sondern auch weithin die von Kirchenjournalisten, die als (leider keineswegs immer christlich-überzeugte) Journalisten vorwiegend über die Ereignisse in der Kirche und Christenheit berichten.

Um die Besonderheit des „missionarischen Stils“ evangelikaler Publizistik zu verstehen, zitieren wir Gerd Rumler aus seinem Buch „Die beste Nachricht der Welt": „Wenn die christliche Gemeinde ihre missionarische Aufgabe erfüllen will, so muß sie lernen, auch die Massenmedien für ihre Aufgaben einzusetzen. "14 An anderer Stelle definiert er:

„Der christliche Journalist hat der vordergründigen Aktualitätsbezogenheit heutiger Meinungsbildung eine christliche Schau entgegenzusetzen, die Zusammenhänge deutlich macht und dem Menschen zeigt, daß er sich in einem aktuellen Geschehen Gottes befindet. Diese prophetische Sicht macht dem Menschen deutlich, wie Gott heute - und das heißt doch nichts anderes als aktuell - mit ihm reden 
und handeln will. Sie trifft ihn in seiner Situation. Sie bietet ihm an, was er wissen muß, um Vergebung zu erlangen, um durch Christus in einen neuen Menschen verwandelt zu werden."15

Vor der Arbeitsgemeinschaft evangelikaler Schriftleiter führte Rumler über das Selbstverständnis des christlichen Journalisten u.a. aus:

„Der Journalist ist - wenn Sie so wollen, mit einem Spezialauftrag versehen Glied der Gemeinde. Und diese kann er nur verstehen als ecclesia viatorum, als Gemeinde unterwegs. Einmal ist dies die einzige Realität von Gemeinde, und zum andern ergibt sich von daher auch die Dynamik unserer publizistischen Arbeit."

Diese so verstandene journalistische Arbeit durchdringt die evangelikale Presse, auch den Stil der einzelnen Beiträge. Allerdings muß dafür auch ein großer Nachteil in Kauf genommen werden: das Nicht-Erreichen der Fernstehenden.

Die missionarischen Zeitschriften beispielsweise wenden sich mit ihren „missionarisch"geschriebenen Berichten (sowohl die Zeitschriften für die innere als auch die für die äußere Mission) in erster Linie an die Treuen im Lande. Der sich dem Glauben entfremdete Mensch wird kaum Zugang finden, weil ihm schon die verwendete Sprache fremd ist, er viele Begriffe auch gar nicht verstehen kann. Andererseits: Er wird nicht mehr dort abgeholt, wo er „zu Hause“ ist.

Hier kann die evangelikale Publizistik viel von den säkularen Massenmedien lernen, die ihre z.T. sehr teuer gewordenen Zeitungen und Zeitschriften auf dem Markt verkaufen müssen. Ohne Lesernähe, d.h. mit Themen, die möglichst viele interessieren, und einer guten, leicht verständlichen Umgangssprache, ist das Ziel nicht zu verwirklichen. Eine breite Themenvielfalt versteht sich für die allgemeine Presse von selbst. Im Gegensatz zu den meisten evangelikalen Zeitschriften, deren Themen-Radius viel enger gesteckt ist.

\section{Anmerkungen}

- Die vollständige Untersuchung erschien unter dem Titel „Die missionarische Stimme Evangelikale Presse in Deutschland“ im Oktober 1981 als Buch im Coprint-Verlag Wiesbaden.

1 Evangelische Presse. Eine Bestandsaufnahme evangelischer Periodika, Frankfurt 1978.

2 Publizistischer Gesamtplan der Evangelischen Kirche in Deutschland, Gütersloh 1979.

${ }^{3}$ Evangelisches Gemeindelexikon, Hrsg. v. Erich Geldbach, Helmut Burkhardt, Kurt Heimbucher, Wuppertal 1978.

${ }^{4}$ Ulrich Betz: Evangelikal. In: Evangelisches Gemeindelexikon, a.a.O., S. 159.

5 Ebd.

6 Ebd.

7 Stimme des Evangeliums, März 1979, S. 7.

8 Ebd.

9 dpa-informationen (Massenmedien-Medienpolitik), v. 3.4.1979.

10 idea v. 4.9.1978.

11 idea v. 19.2.1972.

12 Otmar Schulz: DEZ-gewogen und zu leicht befunden. epd, Kirchliche Presse, Nr. 9/1079. 
14 Gerd Rumler: Die beste Nachricht der Welt, Wuppertal 1978, S. 15.

15 Ebd. S. 72.

16 Gerd Rumler: Das Selbstverständnis des christlichen Journalisten, idea-Dokumentation $14 / 81$, S. 1.

\section{SUMMARY}

The Evangelicals, constituted e.g. by the pietists, biblical fundamentalists and similar groupings - not only are about $40 \%$ of the evangelical Christians in the Federal Republic of Germany, they also have up till now unknown power in the Press. A survey of 144 evengelical periodicals shows that they have about 33 million copies, in fact the number of titles will be higher but to date there is no complete list. Compared with the general evangelical Church press which had, according to a survey in 1976, about 80 titles with a combined circulation of 160 million copies, the evangelical periodicals are in their content missionary and evangelistic orientated, they are mainly financed through gifts and are edited by part-time editors. This evangelical press can be divided into the following groupings: parish bulletins, Mission groups, youth and cooperators periodicals as well as general distribution and devotional, theological and students periodicals. The biggest circulation of these have the publications for general distribution with about 11 million copies. With 33 titles, the mission periodicals are the biggest group by titles, with a total circulation of 4,6 million copies. About one third of the publications do not use any news agency, one other third take the evangelical news service ,idea“, and the remaining third receives the "Evangelischen Pressedienst" (epd) and other agencies like the Catholic News Agency (KNA).

\section{RÉSUMÉ}

Les «évangélicaux» - un mouvement se composant de piétistes, de fondamentalistes bibliques, d'églises libérales et de protestants conservateurs qui se distinguent surtout par l'évangélisation et la mission - ne forment pas seulement environ $40 \%$ des chrétiens évangéliques en Allemangne Fédérale, mais également "une force de presse» jusque-là complètement inconnue. Cela ressort de la première enquête sur la presse «évangélicale» que le journaliste et théologien K. Rüdiger Durth, de Bonn, a entrepris.

A la base de cette enquête, il y a 144 revues «évangélicales qui atteignent un tirage annuel de 33 millions d'exemplaires. Mais le nombre effectif de titres est beaucoup plus élevé. Seulement il n'est pas facile à évaluer car jusqu'à présent, il n'y avait même pas une liste de revues «évangélicales».

Durth comprend la presse (évangélicale» comme partie intégrante de la publicistique évangélique à l'intérieur de laquelle elle forme un groupe propre. A l'opposé de la presse évangélique qui, d'après la dernière enquête de la GEP de 1976, dispose vraisemblablement de plus de 800 titres avec un tirage annuel d'environ 160 millions d'exemplaires, - ici sont comprises également quelques revues "évangélicales» peu nombreuses -, la presse "évangélicale» se distingue par les critères principaux suivants: orientation missionaire, respectivement évangélique, du contenu, financement en grande partie grâce aux dons et par une rédaction à titre de fonction secondaire.

Durth range la presse "évangélicale» en 10 groupes principaux: revues communales, missionaires, de groupes de la jeunesse, de collaborateurs, de distributeurs, de construction, théologiques, estudiantines et diverses. Les revues à distribuer forment le groupe le plus important avec un tirage annuel d'environ 11 millions d'exemplaires. Les revues missionaires, avec 33 titres, forment le plus grand groupe ísolé. Leur tirage annuel s'élève á 4,6 millions d'exemplaires. 


\section{RESUMEN}

Los evangelicalistas - un movimiento integrado por pietistas, fundamentalistas bíblicos, iglesias libres y protestantes conservadores, caracterizados sobre todo por la evangelización y la misión - no sòlo constituyen aproximadamente el 40 por ciento de los cristianos evangélicos de la República Federal de Alemania, sino también una "potencia editorial» totalmente desconocida hasta ahora. Tal conclusión se deduce del primer estudio sobre la prensa evangelicalista, realizado por el periodista y teólogo K. Rüdiger Durth, de Bonn.

El estudio se ocupa de 144 revistas evangelicalistas con una tirada anual global de unos 33 millones de ejemplares. Pero el número total de publicaciones es seguramente muy superior. Es difícil hacer balance pues hasta ahora ni siquiera había una lista de revistas evangelicalistas.

Según Durth la prensa evangelicalista forma parte del publicismo protestante como sector específico. A tenor de la última estadística, realizada en 1976, la prensa protestante cuenta con unos 800 títulos, con una tirada global anual de unos 160 millones de ejemplares, en los que se incluyen algunas revistas evangelicalistas con pequeña tirada. En contraste con la prensa protestante en general, la evangelicalista se caracteriza por los siguientes criterios: concepción misionera o evangelicalista del contenido, financiación a base de limosnas y ahorro de costes mediante redactores no fijos.

Durth articula la prensa evangelicalista en diez grupos principales: revistas destinadas a la comunidad, a la misión, a grupos. a jóvenes, a colaboradores, a distribución, a la consolidación, teológicas, para estudiantes y diversas. El grupo con mayor tirada es el de distribución, con unos once millones de ejemplares. Pero las revistas misionales son las más numerosas: 33 títulos. Su tirada global es de 4,6 millones de ejemplares al año. 